\title{
What Orthopaedic Operating Room Surfaces Are Contaminated With Bioburden? A Study Using the ATP Bioluminescence Assay
}

\author{
Raveesh Daniel Richard MD, Thomas R. Bowen MD
}

Published online: 3 January 2017

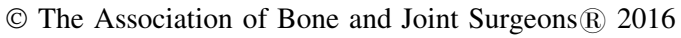

\begin{abstract}
Background Contaminated operating room surfaces can increase the risk of orthopaedic infections, particularly after procedures in which hardware implantation and instrumentation are used. The question arises as to how surgeons can measure surface cleanliness to detect increased levels of bioburden. This study aims to highlight the utility of adenosine triphosphate (ATP) bioluminescence technology as a novel technique in detecting the degree of contamination within the sterile operating room environment.

Questions/Purposes What orthopaedic operating room surfaces are contaminated with bioburden?

Methods When energy is required for cellular work, ATP breaks down into adenosine biphosphate (ADP) and phosphate $(\mathrm{P})$ and in that process releases energy. This
\end{abstract}

\footnotetext{
Each author certifies that he, or a member of his immediate family, has no funding or commercial associations (eg, consultancies, stock ownership, equity interest, patent/licensing arrangements, etc) that might pose a conflict of interest in connection with the submitted article.

All ICMJE Conflict of Interest Forms for authors and Clinical

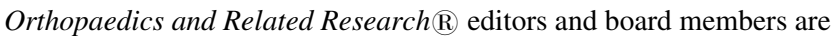
on file with the publication and can be viewed on request.

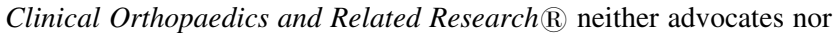
endorses the use of any treatment, drug, or device. Readers are encouraged to always seek additional information, including FDAapproval status, of any drug or device prior to clinical use.

Each author certifies that his institution approved or waived approval for the human protocol for this investigation and that all investigations were conducted in conformity with ethical principles of research.
}

R. D. Richard ( $₫)$, T. R. Bowen

Department of Orthopaedics, Geisinger Medical Center,

100 N Academy Avenue, Danville, PA 17822, USA

e-mail: raveeshrichard@gmail.com process is inherent to all living things and can be detected as light emission with the use of bioluminescence assays. On a given day, six different orthopaedic surgery operating rooms (two adult reconstruction, two trauma, two spine) were tested before surgery with an ATP bioluminescence assay kit. All of the cases were considered clean surgery without infection, and this included the previously performed cases in each sampled room. These rooms had been cleaned and prepped for surgery but the patients had not been physically brought into the room. A total of 13 different surfaces were sampled once in each room: the operating room (OR) preparation table (both pre- and postdraping), OR light handles, Bovie machine buttons, supply closet countertops, the inside of the Bair Hugger ${ }^{\mathrm{TM}}$ hose, Bair Hugger ${ }^{\mathrm{TM}}$ buttons, right side of the OR table headboard, tourniquet machine buttons, the Clarksocket attachment, and patient positioners used for total hip and spine positioning. The relative light units (RLUs) obtained from each sample were recorded and data were compiled and averaged for analysis. These values were compared with previously published ATP benchmark values of 250 to 500 RLUs to define cleanliness in both the hospital and restaurant industries.

Results All surfaces had bioburden. The ATP RLUs (mean $\pm \mathrm{SD}$ ) are reported for each surface in ascending order: the OR preparation table (postdraping; $8.3 \pm 3.4$ ), inside the sterilized pan $(9.2 \pm 5.5)$, the inside of the Bair Hugger $^{\mathrm{TM}}$ hose $(212.5 \pm 155.7)$, supply closet countertops (281.7 \pm 236.7$)$, OR light handles $(647.8 \pm 903.7)$, the OR preparation table (predraping; $1054 \pm 387.5$ ), the Clarksocket attachment $(1135.7 \pm 705.3)$, patient positioners used for total hip and spine positioning (1201.7 \pm 1144.9 ), Bovie machine buttons $(1264.5 \pm 638.8)$, Bair Hugger ${ }^{\mathrm{TM}}$ buttons (1340.8 \pm 1064.1 ), tourniquet machine buttons (1666.5 \pm 2144.9$)$, computer keyboard (1810.8 \pm 929.6), 
and the right side of the OR table headboard $(2539 \pm 5635.8)$.

Conclusions ATP bioluminescence is a novel method to measure cleanliness within the orthopaedic OR and can help identify environmental trouble spots that can potentially lead to increased infection rates. Future studies correlating ATP bioluminescence findings with microbiology cultures could add to the clinical utility of this technology.

Clinical Relevance Surfaces such as the undersurface of the OR table headboard, Bair Hugger ${ }^{\mathrm{TM}}$ buttons, and tourniquet machine buttons should be routinely cleansed as part of an institutional protocol. Although correlation between ATP bioluminescence and clinical infection was not evaluated in this study, it is the subject of future research. Specifically, evaluating microbiology samples taken from these environmental surfaces and correlating them with increased bioburden found with ATP bioluminescence technology can help promote improved surgical cleaning practices.

\section{Introduction}

Healthcare-associated infections often have multiple etiologies, of which cleanliness of hospital surfaces can play a large role [11]. This is perhaps most important in the operating room, where a sterile environment is paramount to decreasing the burden of hospital-acquired morbidity and surgical site infections. Contaminated hospital surfaces greatly contribute to the transmission of healthcareassociated pathogens, including methicillin-resistant Staphylococcus aureus (MRSA), vancomycin-resistant Enterococcus spp (VRE), and Clostridium difficile [19, 21]. In fact, MRSA and VRE can survive for weeks on environmental surfaces in healthcare facilities [4]. Evidence exists demonstrating that when patients enter a room previously occupied by a patient with MRSA, VRE, Acinebacter spp, or $C$ difficile, the new patient is at increased risk for acquiring the infection $[12,19,21]$.

First used in the food industry, adenosine triphosphate (ATP) bioluminescence monitoring has been become broadly applicable in the healthcare setting to provide rapid results regarding hospital cleanliness with improved benefits in the control of surface contamination and application of corrective action against poor hygiene [1, 3]. Because ATP hydrolysis (ATP $\rightarrow$ ADP $+\mathrm{P}_{\mathrm{i}}+$ energy) is inherent to all living organisms, including bacteria, ATP bioluminescence monitoring is a convenient method to visualize localized bioburden on contaminated surfaces. Hospitals have developed numerous cleaning protocols to reduce contamination in the clinical setting. For instance, Boyce et al. measured surface contamination of five high-touch surfaces in patient rooms including bedside rails, overbed tables, television remote controls, toilet seats, and bathroom grab bars [5]. Inadequate cleaning practices were documented by determining aerobic colony counts and by use of an ATP bioluminescence assay [5].

Within orthopaedic surgery, surgical site infection has become an important focus, particularly in total joint arthroplasty. In addition to the Surgical Care Improvement Project (SCIP) guidelines and other recommendations from the American Academy of Orthopaedic Surgeons (AAOS) such as limiting operating room traffic, efforts to reduce the burden of nosocomial orthopaedic infections have become increasingly important. Previous clinical studies and studies in the restaurant industry have established ATP benchmark values of 250 to 500 relative light units (RLUs) to define cleanliness $[13,16]$. To our knowledge, no previous study has evaluated the use of ATP bioluminescence as a measurement of cleanliness in orthopaedic surgery operating rooms.

We therefore asked: Utilizing ATP bioluminescence, what orthopaedic operating room surfaces are contaminated with bioburden?

\section{Patients and Methods}

This was a prospective diagnostic study. Because there was no patient contact in our study, institutional review board exemption was obtained, and an ATP bioluminescence assay kit was used to measure the cleanliness of surfaces in orthopaedic operating rooms.

ATP bioluminescence assay kits essentially consist of chemically impregnated reagent swabs and a luminometer. Specifically, a compartmentalized releasing-buffering agent in the swabs lyse the cell walls of microorganisms, rapidly releasing ATP. Only live cells requiring ATP for energy metabolism release ATP in this process. The luminometer contains the firefly enzyme, luciferase, which produces a simple bioluminescence reaction when it encounters the released ATP [14]. The amount of ATP produced is measured in RLUs with standards set by the manufacturer. Measuring the amount of bioluminescence from an ATP reaction provides a valuable indication of surface cleanliness because the quantity of light generated by the enzyme reaction is directly proportional to the amount of ATP present in the sample. Because bacteria and other living microorganisms produce ATP, the measurement of bioluminescence is indicative of contamination in a sterile environment [13].

After an orthopaedic surgery had been performed, the six operating rooms were cleaned as per routine hospital protocol in preparation for the next case (two adult reconstruction, two trauma, two spine). A cloth composed 
of $80 \%$ rayon, $15 \%$ polypropylene, and $5 \%$ polyester was dampened with hydrated ethyl alcohol at $70 \%(\mathrm{w} / \mathrm{v})$ and then swept over a surface for at least 10 seconds. Before patient entry into the room, ATP bioluminescence swabs ( $3 \mathrm{M}^{\mathrm{TM}}$ Clean-Trace ${ }^{\mathrm{TM}}$ Surface ATP UXL100; 3 M Corporation, St Paul, MN, USA) were used to take samples of areas in the operating room that were on a spectrum of dirty to sterile after terminal cleaning. "Dirty" surfaces were those considered to be inadequately cleaned between cases as a result of either inattention by environmental service staff (ie, Bair Hugger ${ }^{\mathrm{TM}}$ buttons/hose [3 M Corp.]), surfaces without direct contact with patients (ie, operating room [OR] shelves), or surfaces that routinely were used by OR personnel that were not scrubbed into the procedure (ie, computer keyboards).

The swabs were then analyzed with the use of the handheld luminometer $\left(3 \mathrm{M}^{\mathrm{TM}}\right.$ Clean-Trace ${ }^{\mathrm{TM}} \mathrm{NG}$ Luminometer Version 3.0 [NGi]) that measured the amount of bioburden for the given area that was swabbed. The amount of ATP, both microbial and nonmicrobial, was quantified and expressed as RLUs. This number was recorded and compared with thresholds for contamination set by the hospital industry standard of $>500$ RLUs $[7,13,16]$. In addition, we utilized published acceptability limits for kitchen surfaces used in the restaurant industry of $<400$ RLUs, published by Osimani et al [15]. In this study, sampled surfaces that were above the restaurant industry standard of 400 RLUs and certainly over previously published hospital standards of 500 RLUs were considered contaminated in the sterile OR environment. As previously published, the ATP bioluminescence linearly represents the degree of bioburden and is repeatable in its readings, although some spread/scatter in RLU measurements is expected [14].

Two control points were chosen based on the assumption of bioburden that these surfaces typically carry. The "sterile" control was the inside of a sterilized pan, whereas the "dirty" control was the keyboard of one of the OR computers. Additionally, 11 different surfaces with highvolume contact were tested for ATP bioluminescence: the OR preparation table (both pre- and postdraping), OR light handles, Bovie machine buttons, supply closet countertops, the inside of the Bair Hugger ${ }^{\mathrm{TM}}$ hose, Bair Hugger ${ }^{\mathrm{TM}}$ buttons, right side of the OR table headboard, tourniquet machine buttons, the Clark-socket attachment, and patient positioners used for total hip and spine positioning. These 13 points of interest were tested in a total of six orthopaedic operating rooms for a total of 78 data points.

\section{Results}

All surfaces had bioburden (Table 1; Fig. 1). The ATP RLUs (mean $\pm \mathrm{SD}$ ) are reported for each surface in ascending order: the OR preparation table (postdraping; $8.3 \pm 3.4$ ), inside the sterilized pan $(9.2 \pm 5.5)$, the inside of the Bair Hugger $^{\mathrm{TM}}$ hose $(212.5 \pm 155.7)$, supply closet countertops (281.7 \pm 236.7$)$, OR light handles $(647.8 \pm 903.7)$, the OR preparation table (predraping; $1054 \pm 387.5$ ), the Clarksocket attachment $(1135.7 \pm 705.3)$, patient positioners used for total hip and spine positioning (1201.7 \pm 1144.9$)$, Bovie machine buttons $(1264.5 \pm 638.8)$, Bair Hugger ${ }^{\mathrm{TM}}$ buttons (1340.8 \pm 1064.1$)$, tourniquet machine buttons (1666.5 \pm 2144.9$)$, computer keyboard (1810.8 \pm 929.6),

Table 1. Degree of bioburden on orthopaedic operating room surfaces measured in relative light units (RLUs)

\begin{tabular}{|c|c|c|c|c|}
\hline OR surface & Mean & SD & Minimum & Maximum \\
\hline Inside sterilized pan & 9.2 & 5.5 & 5 & 20 \\
\hline OR preparation table (predrape) & 1054 & 387.5 & 403 & 1562 \\
\hline OR preparation table (postdrape) & 8.3 & 3.4 & 5 & 14 \\
\hline OR light handles & 647.8 & 903.7 & 84 & 2456 \\
\hline Bovie machine buttons & 1264.5 & 638.8 & 366 & 2278 \\
\hline Supply closet countertops & 281.7 & 236.7 & 83 & 677 \\
\hline Inside Bair Hugger ${ }^{\mathrm{TM}}$ hose & 212.5 & 155.7 & 72 & 423 \\
\hline Bair Hugger ${ }^{\mathrm{TM}}$ buttons & 1340.8 & 1064.1 & 278 & 2880 \\
\hline Right side of OR table headboard & 2539 & 5635.8 & 142 & 14,042 \\
\hline Tourniquet machine buttons & 1666.5 & 2144.9 & 453 & 5994 \\
\hline Clark-socket attachment & 1135.7 & 705.3 & 273 & 2159 \\
\hline Patient positioner & 1201.7 & 1144.9 & 296 & 3428 \\
\hline Computer keyboard & 1810.8 & 929.6 & 297 & 2588 \\
\hline
\end{tabular}

$\mathrm{OR}=$ operating room. 


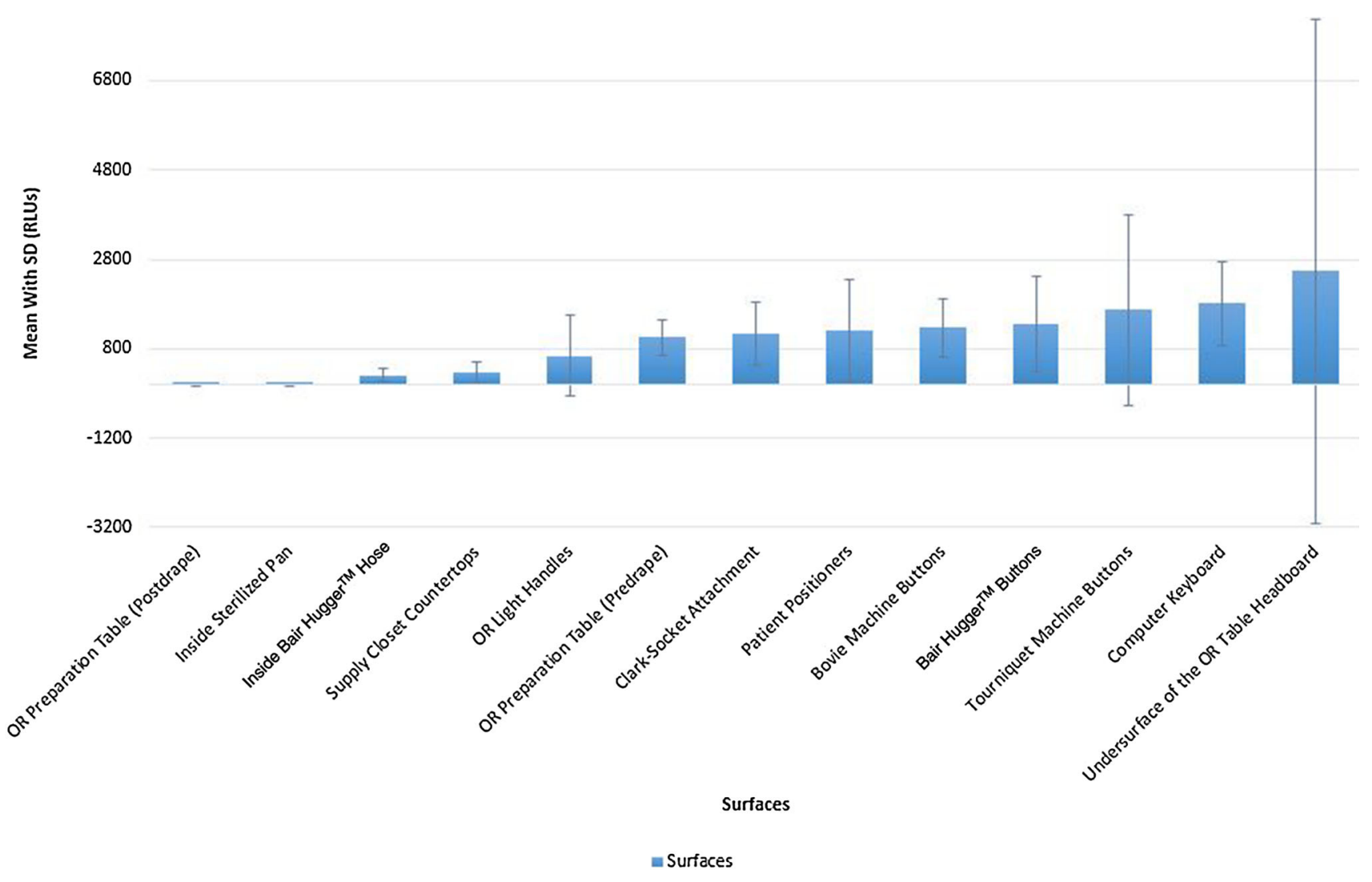

Fig. 1 Degree of bioburden on orthopaedic OR surfaces as measured in RLUs.

and the right side of the OR table headboard $(2539 \pm 5635.8)$. Overall, the surface with the cleanest surface was the OR preparation table postdraping (average 8 RLUs) with the dirtiest surface being the right side of the OR table headboard (average 2539 RLUs).

\section{Discussion}

Contaminated OR surfaces can increase the risk of orthopaedic infections, particularly after procedures in which hardware implantation and instrumentation are used. The question then arises as to how surgeons can measure surface cleanliness to detect increased levels of bioburden. This study aims to highlight the utility of ATP bioluminescence technology as a useful technique in detecting the degree of contamination within the sterile OR environment. Our results demonstrate that several surfaces that are considered "clean" within the OR are in fact not as clean as one may think. Surfaces such as the undersurface of the OR table headboard and machine buttons should be routinely cleaned as a part of the routine OR cleaning protocol to reduce the amount of OR bioburden.

Limitations of ATP bioluminescence testing do exist. It must be stated that increased RLU measurements do not directly correlate to increased clinical infection rates. Still, some authors have demonstrated evidence that when patients enter a room previously occupied by a patient with MRSA, VRE, Acinebacter spp, or $C$ difficile, and where environmental surfaces have not been thoroughly cleaned, the new patient is at increased risk for acquiring the infection [12, 19]. This concept can be applied to the OR. Theoretically, there is an increased risk of nosocomial infection in OR environments that have not been adequately decontaminated in between cases, further highlighting the utility of this present study. Additionally, it has been proposed that organic debris can account for approximately $66 \%$ of ATP on surfaces [10]. Thus, ATP assays can be oversensitive and can potentially overestimate the degree of bacterial bioburden on surfaces. Some studies have highlighted a lack of a true correlation between ATP readings and aerobic colony counts, noting that additional environmental factors such as residual detergent/disinfectants, poor surface conditions, and ammonium cleaning compounds can highly increase or decrease ATP readings [6, 7, 10, 15, 17]. Conversely, falsenegative ATP bioluminescence results have been reported in surfaces cleaned with bleach-based disinfectants [9, 18, 20]. Perhaps the biggest limitation to ATP bioluminescence assay kits is the poor detection of Gram- 
negative bacteria. An animal study by Turner et al. demonstrated that ATP bioluminescence detection of Gram-negative bacteria improved with sonication, suggesting that the buffer in the assay incompletely lyses the cell walls of Gram-negative bacteria [18, 20]. Sonication, however, did not improve the detection of $S$ aureus, indicating that the chemical reagents readily lyse the cell walls of Gram-positive bacteria.

Routine visual inspection has repeatedly been shown to underestimate the degree of bioburden in the healthcare setting $[8,13,16]$. The measurement of organic ATP on surfaces using a luciferase assay and luminometer has been used in the food preparation industry for more than 30 years [11]. Although sensitivity varies between commercially available systems, ATP benchmark values of 250 to 500 RLUs have been used to define cleanliness with very low readings typically associated with low aerobic colony counts $[13,16]$. Conversely, very high RLU readings can represent viable bioburden, organic debris, or a combination of both [7]. Using a benchmark of 100 RLUs, Anderson et al. found that $84 \%$ (37 of 44) items in a surgical ward exceeded this standard and were considered contaminated [2]. Similarly, other studies have raised concerns regarding the standards of surface cleanliness in the hospital environment $[8,16,19]$. If we are to compare our study results with the criteria set forth by the restaurant industry, as outlined previously, the only surfaces in our ORs that would be considered "clean" are the OR preparation table (postdraping), the inside of a sterilized pan, the inside of the Bair Hugger ${ }^{\mathrm{TM}}$ hose, and the supply closet countertops. This is frankly alarming. Surfaces such as OR light handles, the Clark-socket attachment, patient positioners used for total hip and spine positioning, and the right side of the OR table headboard, to name a few, would not even pass restaurant standards so why should this be considered appropriate for the sterile OR environment? It is this precise question that our study aims to highlight. The near $\times 100$ variance of RLU measurements in this study can be explained by the lack of routine cleaning of the surfaces with the highest RLU measurements. This was reproducible throughout the six ORs tested and implies that better cleaning practices need to be instituted to address this oversight. Per Sherlock et al. [18], an amendment in cleaning practice at their institution led to a decrease in ATP bioburden in a hospital setting (average of 612 RLUs precleaning versus 375 RLUs postcleaning).

Despite cleaning, we found bioburden levels that would be concerning even in the food industry [13, 16] on OR light handles, the OR preparation table (predraping), the Clark-socket attachment, patient positioners used for total hip and spine positioning, Bovie machine buttons, Bair Hugger $^{\mathrm{TM}}$ buttons, tourniquet machine buttons, computer keyboards, and the right side undersurface of the OR table headboard. Although not all these surfaces may physically make contact with the patient, the presence of contamination within a sterile OR environment could potentially lead to further contamination. It is conceivable that the surgeon, nurse, or anesthesiologist could transfer bacteria from one surface to another or to the patient simply by touching the Bair Hugger ${ }^{\mathrm{TM}}$ buttons, tourniquet machine, or light handles and not washing their hands directly afterward. This suggests that more attention needs to be paid to those surfaces during the terminal cleaning and sterilization processes between surgical procedures. Griffith et al. [10] demonstrated that $61 \%$ of surfaces within the OR environment were considered unacceptable in cleanliness. Utilizing established ATP benchmarks of 500 RLUs and microbiology surface samples with less than 2.5 colony forming units (CFUs) $/ \mathrm{cm}^{2}$, the authors demonstrated that visual inspection alone would grossly underestimate the level of cleanliness in the OR suite. Although a direct correlate between these established benchmarks and clinical infection has not been shown, it is conceivable that contamination above these thresholds could potentially increase the risk of surgical site infection. This issue seems especially important in orthopaedic surgery, where hardware implantation is commonplace and any increased potential for bioburden would seem to result in a serious concern about infection. Our results demonstrate that multiple surfaces in the OR were contaminated. It is conceivable that when OR personnel clean in between cases, these surfaces are often left untouched and do not get wiped down because they do not routinely have direct contact with patients or within the surgical field. However, it must be noted that, at our institution, the right side of the OR table headboard is often an area that is used for placing the suction tip used by anesthesia to clear secretions during induction/intubation and postextubation. Considering the bioburden in oral flora, this is a critical part of the OR that often goes uncleaned and can potentially contribute to increased surgical site infections [4, 12, 19].

It is assuring that surfaces expected to be clean such as the prepped OR table and sterilized pans were indeed near negligible with bioburden. It was somewhat surprising that although the inside of the Bair Hugger ${ }^{\mathrm{TM}}$ hose does not routinely get cleaned at our institution, the degree of bioburden was relatively small compared with other OR surfaces. Perhaps this finding can be attributed to limited exposure to the environment, especially because surfaces that were routinely touched by hand (computer keyboards, tourniquet machine buttons, Bair Hugger ${ }^{\mathrm{TM}}$ buttons, patient positioners, and Clark-socket attachments) had the highest overall RLUs on bioluminescence testing.

ATP bioluminescence is a novel method to measure cleanliness within the orthopaedic OR and can help identify environmental trouble spots that can potentially lead to 
increased infection rates. Surfaces such as the undersurface of the OR table headboard, Bair Hugger ${ }^{\mathrm{TM}}$ buttons, and tourniquet machine buttons should be routinely cleansed as part of an institutional protocol. Although a direct correlation between ATP bioluminescence and clinical infection was not evaluated in this study, it is the subject of future research. Specifically, evaluating microbiology samples taken from these environmental surfaces and correlating them with increased bioburden found with ATP bioluminescence technology can help promote improved surgical cleaning practices. Improved cleanliness of hospital surfaces can help reduce risk of nosocomial infections, particularly related to surgery $[4,12,16,19]$. Although our results are in some ways encouraging, they also reveal that many surfaces in a supposedly "sterile" environment often go uncleaned. Within orthopaedic surgery, where routine implantation of metallic prostheses and hardware predominates, reducing the risk of environmental contamination is imperative.

Acknowledgments We thank Christopher Beauchamp MD (Mayo Clinic Arizona, Phoenix, AZ, USA) for his recommendations and contribution to the study.

\section{References}

1. Aiken ZA, Wilson M, Pratten J. Evaluation of ATP bioluminescence assays for potential use in a hospital setting. Infect Control Hosp Epidemiol. 2011;32:507-509.

2. Anderson RE, Young V, Stewart M, Robertson C, Dancer SJ. Cleanliness audit of clinical surfaces and equipment: who cleans what? J Hosp Infect. 2011;78:178-181.

3. Aycicek H, Oguz U, Karci K. Comparison of results of ATP bioluminescence and traditional hygiene swabbing methods for the determination of surface cleanliness at a hospital kitchen. Int J Hyg Environ Health. 2006;209:203-206.

4. Boyce JM. Environmental contamination makes an important contribution to hospital infection. J Hosp Infect. 2007;65:50-54.

5. Boyce JM, Havil NL, Dumigan DG, Golebiewski M, Balogun O, Rizvani R. Monitoring the effectiveness of hospital cleaning practices by use of an adenosine triphosphate bioluminescence assay. Infect Control Hosp Epidemiol. 2009;30:678-684.

6. Brown E, Eder AR, Thompson KM. Do surface and cleaning chemistries interfere with ATP measurement systems for monitoring patient room hygiene? J Hosp Infect. 2010;74:193-195.
7. Carling PC, Bartley JM. Evaluating hygienic cleaning in health care settings: what you do not know can harm your patients. Am J Infect Control. 2010;38:S41-S50.

8. Cooper RA, Griffith CJ, Malik RE, Obee P, Looker N. Monitoring the effectiveness of cleaning in four British hospitals. Am J Infect Control. 2007;35:338-341.

9. Green TA, Russell SM, Fletcher DL. Effect of chemical cleaning agents and commercial sanitizers on ATP bioluminescence measurements. J Food Prot. 1999;62:86-90.

10. Griffith CJ, Cooper RA, Gilmore J, Davies C, Lewis M. An evaluation of hospital cleaning regimes and standards. J Hosp Infect. 2000;45:19-28.

11. Hota B. Contamination, disinfection, and cross-colonization: are hospital surfaces reservoirs for nosocomial infection? Clin Infect Dis. 2004;39:1182-1189.

12. Huang SS, Datta R, Platt R. Risk of acquiring antibiotic-resistant bacteria from prior room occupants. Arch Intern Med. 2006;166:1945-1951.

13. Lewis T, Griffith C, Gallo M, Weinbren M. A modified ATP benchmark for evaluating the cleaning of some hospital environmental surfaces. J Hosp Infect. 2008;69:156-163.

14. Omidbakhsh N, Ahmadpour F, Kenny N. How reliable are ATP bioluminescence meters in assessing decontamination of environmental surfaces in healthcare settings? PLoS One. 2014;9: e99951.

15. Osimani A, Garofalo C, Clementi F, Tavoletti S, Aquilanti L. Bioluminescence ATP monitoring for the routine assessment of food contact surface cleanliness in a university canteen. Int $J$ Environ Res Public Health. 2014;11:10824-10837.

16. Poulis JA, de Pijper M, Mossel DA, Dekkers PP. Assessment of cleaning and disinfection in the food industry with the rapid ATPbioluminescence technique combined with the tissue fluid contamination test and a conventional microbiological method. Int $J$ Food Microbiol. 1993;20:109-116.

17. Sharma A, Saurabh K, Yadav S, Jain SK, Parmar D. Expression profiling of selected genes of toxication and detoxication pathways in peripheral blood lymphocytes as a biomarker for predicting toxicity of environmental chemicals. Int J Hyg Environ Health. 2013;216:645-651.

18. Sherlock O, O'Connell N, Creamer E, Humphreys H. Is it really clean? An evaluation of the efficacy of four methods for determining hospital cleanliness. J Hosp Infect. 2009;72:140-146.

19. Stannard CJ, Gibbs PA. Rapid microbiology: applications of bioluminescence in the food industry-a review. $J$ Biolumin Chemilumin. 1986;1:3-10.

20. Turner DE, Daugherity EK, Altier C, Maurer KJ. Efficacy and limitations of an ATP-based monitoring system. J Am Assoc Lab Anim Sci. 2010;49:190-195.

21. Weber DJ, Anderson D, Rutala WA. The role of the surface environment in healthcare-associated infections. Curr Opin Infect Dis. 2013;26:338-344. 